\title{
EL ABORDAJE DE LA PEDAGOGÍA HOSPITALARIA EN EL CONTEXTO VENEZOLANO
}

\section{The approach of Hospital Pedagogy in the Venezuelan context}

\author{
Marian SERradas Fonseca \\ Universidad Nacional Abierta de Venezuela, Centro Local Yaracuy \\ Correo electrónico:mserradas@una.edu.ve; mserradas@hotmail.com \\ Recepción: 28 de noviembre de 2016 \\ Envío a informantes: 5 de diciembre de 2016 \\ Aceptación definitiva: 20 de febrero de 2017
}

Resumen: Este documento tiene por objeto representar la realidad de la Pedagogía Hospitalaria en Venezuela. Su propósito es ofrecer una visión histórica, en primer lugar, de los hechos más relevantes que permitieron el surgimiento, desarrollo y la consolidación de la Pedagogía Hospitalaria en Venezuela, así como la implementación y puesta en marcha de las primeras aulas hospitalarias. Luego, aborda la formación docente en Pedagogía Hospitalaria, continúa con la investigación en el avance de esta modalidad educativa en nuestro contexto, así como la descripción de programas y proyectos que se realizan en el país en beneficio de la población hospitalizada y la presentación de algunas consideraciones finales.

Palabras clave: Pedagogía Hospitalaria; Aula Hospitalaria; Docente Hospitalario; Niños Hospitalizados.

Aвstract: This document is intended to represent the reality of Hospital Pedagogy in Venezuela. Its purpose is to provide a historical view first, of the most significant events that allowed the emergence, development and consolidation of Hospital Pedagogy in Venezuela, as well as the implementation and commissioning of the first hospital wards. Then, addressing teacher training in Hospital Pedagogy, he continues to research in the advancement of this type of education in our context, as well as the description of programs and projects conducted in the country for the benefit of the hospitalized population and the presentation of some final considerations.

Key words: Hospital Pedagogy; Hospital Classroom; Teaching Hospital; Hospitalized Children.

Una prueba de lo acertado de la intervención educativa es la felicidad del niño. 


\section{Consideraciones generales}

$\mathrm{L}$

a Pedagogía Hospitalaria en Venezuela, articulada bajo la modalidad de la Educación Especial, surge como respuesta a la necesidad de atención y continuidad en el proceso de enseñanza y aprendizaje, del niño y adolescente que, a causa de la enfermedad y el proceso de enfermar, presentan un cambio brusco y repentino en su rutina de vida que repercutirá en su escolaridad.

En la perspectiva que se adopta en este documento se considera la definición de Violant, Molina y Pastor (20II) sobre la Pedagogía Hospitalaria como la acción pedagógica que se desarrolla durante los procesos de enfermedad para dar respuesta a las necesidades biopsicosociales derivadas de dicha situación, con el fin de mejorar el bienestar y la calidad de vida, garantizando los derechos con relación a la función educativa.

De este modo, en Venezuela, la labor pedagógica hospitalaria se ha venido desarrollando de manera espontánea, de modo similar a sus comienzos en los países europeos. Aun cuando en su haber histórico, y según Cardone y Monsalve (20IO), este tipo de intervención en el país cuenta con una experiencia de más de cuarenta años, pareciera que es apenas en este momento cuando se comienza a tomar conciencia de su importancia, lo que obliga a poner sobre el tapete los logros y aciertos que en el camino se han ido suscitando.

Dentro de estos aciertos, es importante resaltar que, desde el año 2007, Venezuela forma parte de la Red Latinoamericana y del Caribe por el Derecho a la Educación de los niños, niñas y jóvenes en situación de hospitalización y/o en tratamiento (REDLACEH), ocupando en la actualidad el cargo de la vicepresidencia, esto ha permitido divulgar el trabajo realizado por docentes venezolanos en Latinoamérica y Europa, así como el intercambio de información sobre buenas prácticas realizadas en el resto de países sobre la Pedagogía Hospitalaria.

REDLACEH es una organización internacional sin fines de lucro, que tiene como eje central de su trabajo el Derecho a la Educación, particularmente el velar por el efectivo derecho a la educación de los niños y jóvenes hospitalizados o en tratamiento de América Latina y el Caribe.

Ahora bien, en Venezuela existe una plataforma legal para garantizar los derechos de los niños y adolescentes a través de la Ley Orgánica para la Protección del niño, niña y del adolescente (LOPNA), sin embargo, las necesidades de la población en situación de salud disminuida han sido poco visibilizadas. Con la entrada en vigencia de la LOPNA, inspirada en la Convención Internacional sobre los Derechos del Niño, donde se establece un novedoso sistema de protección de los derechos del niño y del adolescente, se recogen algunos artículos que persiguen la proclamación de la asistencia médica de los menores, como el hecho de reconocer uno de los derechos del niño hospitalizado, que es el de poder permanecer junto a sus padres durante su estancia en el centro de salud público o privado o asegurar la permanencia de tiempo completo de al menos de uno de los padres.

En este sentido, organizaciones como el Círculo de Investigaciones y Estudios con el Psicoanálisis (CIEP) de Venezuela elaboraron una propuesta sobre los Derechos de los niños y adolescentes hospitalizados, que recoge las necesidades de los pequeños pacientes que deben ser atendidas en los centros hospitalarios, necesidades éstas derivadas de la propia enfermedad, de las relacionadas con los procedimientos médicos 
que se han de practicar, a las vinculadas con la estructura y organización del hospital y las asociadas a las relaciones personales. Esta propuesta contiene veintidós (22) derechos de los niños pacientes, entre los cuales se pueden destacar:

- Tengo derecho a ser llamado por mi nombre.

- Tengo derecho a ser tratado con educación y respeto.

- Tengo derecho a que se me alivie el dolor.

- Tengo derecho a recibir información adaptada a mi edad y desarrollo mental sobre los tratamientos que se me van a aplicar.

- Tengo derecho a ser hospitalizado junto a otros niños.

- Tengo derecho a que la hospitalización sea lo más breve posible.

- Tengo derecho a continuar mi educación y recreación dentro del hospital.

- Tengo derecho a permanecer en un espacio limpio y cuidado durante la hospitalización.

- Tengo derecho a estar acompañado de alguno de mis padres durante la enfermedad.

- Tengo derecho a la vida sin discriminación alguna fundada en motivos de color, sexo, edad, idioma, pensamiento, religión, procedencia, discapacidad o enfermedad.

- Tengo derecho a ser feliz.

De igual manera, en el año 2014 en la ciudad de Panamá, en el contexto de la xxi Reunión de la Comisión de Educación, Cultura, Ciencia, Tecnología y Comunicación, del Parlamento Latinoamericano, se aprobó el articulado definitivo que compone la Ley Marco para la Pedagogía Hospitalaria en América Latina y el Caribe, el cual fue aprobado por unanimidad.

La Ley Marco de Pedagogía Hospitalaria es el resultado de un trabajo conjunto de los miembros e investigadores de REDLACEH, de la que como se mencionó con anterioridad Venezuela forma parte, el cual se inició a partir del año 2009 con la redacción de la Carta de los derechos del niño y joven hospitalizado o en tratamiento en el ámbito de la educación, carta que fue aprobada por esta misma Comisión en noviembre del 2013.

Se hace necesario referir que no todos los hospitales públicos de Venezuela brindan atención pedagógica, a pesar de que resulta altamente necesaria esta intervención desde un marco institucional y profesional. Es evidente entonces que sería muy importante, tal como sostiene Serradas (2008), que en Venezuela se asumiera, como ya se ha hecho en otros países, una Carta de los Derechos del Niño Hospitalizado, acorde con las situaciones y particularidades, así como con las posibilidades reales del sector salud.

\section{Antecedentes y evolución de las Aulas Hospitalarias en Venezuela}

Para el año I948 se registra la primera experiencia sistematizada de Pedagogía Hospitalaria en Venezuela y es en el «Aula Jardín Dra. Lya Imber» ubicada en el Pabellón de Niños del Hospital Central de Valencia, dependiente para el momento del Ministerio de Sanidad y Asistencia Social.

Desde el año 1969 y hasta la actualidad existen experiencias registradas de la atención pedagógica al niño y adolescente hospitalizado, en aulas anexas a los hospitales 
generales y hospitales de especialidades pediátricas, que surgen como jardines de infancia, escuelas unitarias, aulas hospitalarias, centros educativos hospitalarios y espacios psicorrecreativos, en los que docentes provenientes de las diferentes áreas de la educación ofrecen apoyo pedagógico durante la estancia del niño y adolescente en el hospital.

Para el año 1976, el Ministerio de Educación, bajo la Dirección de Educación Especial, materializa la propuesta de atención de escolares con impedimentos físicos, bajo la figura de Aulas Anexas a hospitales, Escuelas Unitarias Hospitalarias atendiendo a la población de niños y adolescentes hospitalizados. Paralelamente se encuentran en funcionamiento los Centros Educativos Hospitalarios desde el año 1979 bajo la dirección para entonces del Consejo Venezolano del Niño, actualmente Fundación del Niño Simón; y Espacios Psicorrecreativos bajo la responsabilidad de fundaciones y ONG.

En atención a lo expuesto cabe destacar que para el año de 1998 el Documento de Conceptualización y Política de la Atención Educativa de las Personas con Impedimentos Físicos del Ministerio de Educación, Cultura y Deportes establece que el modelo de atención educativa de la persona con limitaciones físico-motoras debe ser integral, continuo, permanente, sistemático y articulado con los diferentes subsistemas del sistema educativo a fin de garantizar el ingreso, prosecución y culminación del año escolar de los educandos que se encuentran hospitalizados. Por tanto, hasta el is de agosto del año 2009, fecha en la que se promulgó la nueva Ley Orgánica de Educación, las aulas hospitalarias estuvieron canalizadas a través de la Atención Educativa de las Personas con Impedimentos Físicos, considerando por ello a los niños y adolescentes hospitalizados como personas con limitaciones físico-motoras.

Según el documento emanado por el Ministerio de Educación y Deportes, titulado: La Pedagogía Hospitalaria en el Marco del Sistema Educativo Bolivariano, presentado por John (2006), la misión de las Aulas Hospitalarias en el país está dirigida a garantizar la atención educativa integral a niños y jóvenes que asistan al Hospital, para asegurar el inicio, prosecución y culminación escolar en el sistema educativo, mantener comunicación con todas las personas con las que están vinculados: padres, familiares, equipos de salud; además de establecer y mantener contacto con las escuelas de origen, con la finalidad de hacer un seguimiento a su desenvolvimiento e integrarlo a ella tan pronto como sea posible.

Entre los Objetivos Generales de las Aulas Hospitalarias, se pueden mencionar: proporcionar atención educativa integral a la población de niños y adolescentes hospitalizados, para asegurar la continuidad del proceso de aprendizaje; favorecer, a través de un clima de participación e interacción, la integración, evitando procesos de angustia y aislamiento, y fomentar la utilización formativa del tiempo libre en el hospital, programando actividades de ocio de carácter recreativo y educativo.

Los Objetivos Específicos de las Aulas Hospitalarias están divididos en Pedagógicos, Psicológicos y Sociales.

Objetivos Pedagógicos:

- Favorecer la continuidad en el proceso educativo y garantizar su incorporación a la vida escolar regular.

- Generar el interés por aprender y ejercitar el hábito de trabajar.

- Introducir la ocupación constructiva. 
Objetivos Psicológicos:

- Favorecer la mejor adaptación posible de los niños y adolescentes al nuevo ambiente.

- Comprender lo que está sucediendo y por qué.

- Disminuir las vivencias negativas, tales como: ansiedad, miedo.

- Proporcionar información adecuada, responder a sus inquietudes empleando un lenguaje sencillo.

Objetivos Sociales:

- Crear un ambiente lo más cercano a su vida diaria.

- Propiciar un contexto que garantice relaciones de confianza y seguridad.

- Establecer y dar origen a distintas situaciones de relación que favorezcan las relaciones interpersonales entre los niños y los adultos.

- Dar respuestas a sus necesidades sociales desde el reconocimiento de su instancia local.

La población atendida en las aulas hospitalarias es flotante, comprendiendo desde recién nacido hasta los i8 años, referidos a hospitalización por diferentes condiciones de salud. El proceso de atención se desarrolla de acuerdo a una rutina diaria establecida entre las 7 a $\mathrm{I} 2 \mathrm{am}$, donde se cumplen actividades de tipo administrativo de la docente, planificación de las actividades del día, así como selección de los materiales a utilizar, recorrido por las habitaciones, entrega de material y actividades con los niños que no pueden asistir al aula hospitalaria, trabajo directo con los niños en el aula, con participación de los representantes. Asimismo, en la planificación se contempla la realización de círculos de estudio o conversatorios con los padres y representantes, para abordar temas específicos y de su interés.

La planificación se estructura a través de la metodología por Proyectos tomando como referencia al Currículo Básico de Educación Inicial o Básica propuesto por el Ministerio de Educación, el cual incluye el Proyecto Integral Comunitario y el Proyecto de Aula, captando los intereses de la población asistente.

En relación a la población asistente al aula hospitalaria, el contacto con los docentes de la escuela de donde proviene el educando, se realiza a través de la madre o representante del niño o adolescente, cuando éste pasa un tiempo hospitalización mayor de quince días. A través de comunicación escrita, se establece la articulación. Al finalizar la hospitalización se hace entrega de un informe que le permite conocer la situación del niño o adolescente y recomendaciones a seguir.

En relación al personal, está conformado por docentes de Educación Especial, de Educación Preescolar o Inicial, Integral y en algunos equipos se cuenta con la presencia del trabajador social. El resto del equipo interdisciplinario está conformado por otros especialistas que se desempeñan dentro del Hospital, como pueden ser: psicólogo, psiquiatra, terapista de lenguaje, fisioterapeuta y voluntarios, quienes apoyan y complementan la labor docente en el contexto hospitalario. Los docentes realizan su trabajo pedagógico en el aula, en la propia habitación o sala de hospitalización.

Cabe destacar que, en el año 2012 surge una nueva denominación de estos recintos llamados Aulas Hospitalarias transformándose en Espacios Educativos en el Contexto Hospitalario. 
En la actualidad, en Venezuela existen más de 78 espacios en Hospitales Generales y Hospitales de Especialidades Pediátricas destinados a la atención educativa integral del niño y adolescente hospitalizado.

Por último, es conveniente acotar que muchas de estas aulas hospitalarias comenzaron su funcionamiento en el país gracias a la decisión de algunas asociaciones, voluntariado, por la acción de algunos profesionales, organismos estadales y municipales, preocupados por la falta de escolaridad de los pacientes pediátricos. Sin embargo, las mismas no logran cubrir los objetivos generales que a nivel internacional se plantean respecto a su funcionamiento, entre los cuales, la continuidad escolar, entendida como la promoción al grado sucesivo si éste fuera el caso. Ya que la mayoría de las aulas hospitalarias han centrado su quehacer en el apoyo pedagógico a través de lo lúdico y la recreación y en pocas ocasiones se consigue articular realmente al alumno hospitalizado con el sistema de educación formal.

\section{Formación docente en Pedagogía Hospitalaria}

Los docentes que laboran y están a cargo de los espacios educativos en contextos hospitalarios en Venezuela son egresados de diferentes Institutos de Educación Universitaria y Universidades en las que se imparte la carrera de Educación en las diferentes menciones y/o especialidades. Estos docentes, tal como afirma Hermo (2013), no poseen formación curricular formal dentro del pensum de estudios, tanto en las instituciones públicas como privadas. Es la experiencia en la praxis lo que permite desarrollar las distintas actividades y competencias, desde los enfoques formativo, instructivo y psicopedagógico.

En tal sentido, desde el año 2009 y de forma ininterrumpida, la Escuela de Educación de la Universidad Central de Venezuela oferta la asignatura Pedagogía Hospitalaria, de carácter electivo, adscrita al Departamento de Psicología Educativa, sobre la atención pedagógica a niños hospitalizados o en tratamiento ambulatorio. La inclusión en los programas de formación docente en esta casa de estudios de asignaturas y afines referentes a la Pedagogía Hospitalaria nace de la necesidad de brindar a los futuros docentes conocimientos y referentes a la atención y educación de los niños y adolescentes en situación de enfermedad y/u hospitalizados, con la finalidad de proporcionar herramientas básicas de carácter teórico-práctico referentes a la atención pedagógica de niños y adolescentes hospitalizados o en tratamiento ambulatorio.

Según Rodríguez (2012), el objetivo de este plan curricular sobre Pedagogía Hospitalaria es el de propiciar el desarrollo de estrategias para elaborar programas educativos basados en las necesidades educativas especiales asociadas a la enfermedad y al proceso de hospitalización infantil. Al finalizar el programa, los estudiantes tendrán la oportunidad de visitar una Escuela, Aula o Centro Educativo Hospitalario. Dicha visita tiene como propósito vincular al estudiante con el ambiente de dichos lugares y el trabajo que se realiza con los pacientes pediátricos hospitalizados. En esta visita, el estudiante tendrá la posibilidad de conocer las instalaciones hospitalarias, así como del Aula, Escuela o Centro Educativo Hospitalario, conocer y vincularse con el trabajo del equipo y compartir con los niños hospitalizados.

De igual manera, desde el año 2010 el Instituto Pedagógico de Caracas viene ofertando la asignatura optativa Abordaje Pedagógico del infante en situación de 
hospitalización, adscrita al Departamento de Educación Especial. Este curso de naturaleza teórico-práctica va destinado a proporcionar en el estudiante la adquisición de conocimientos y el desarrollo de habilidades para la planificación y ejecución de situaciones didácticas, a través de la selección de métodos y técnicas para el abordaje pedagógico de niños en situación de hospitalización, que permita dar garantía del reingreso satisfactorio al sistema educativo regular, centrado en los fundamentos de la pedagogía hospitalaria.

Según Lizardo (20Io), el objetivo fundamental de esta asignatura es el de proveer oportunidades para el diseño de situaciones didácticas a partir del análisis del entorno y de las características del infante en situación de hospitalización, lo que posteriormente permitirá el diseño y ejecución de un plan de abordaje pedagógico, centrado en los intereses y necesidades de los pequeños pacientes. En la actualidad esta unidad curricular se continúa ofertando en el nuevo pensum en régimen de libre elección, sin embargo, su contenido curricular abarca mucho más que la atención educativa en el hospital.

Dentro de esta línea de acción el Instituto Pedagógico de Caracas cuenta además con el Proyecto de Servicio Comunitario titulado Payasos de Hospital: Calidad de Vida en Acción, dirigido a todas las especialidades educativas que se imparten en este instituto de educación superior, como una forma de contribuir a mejorar la calidad de vida de los niños y adolescentes y sus familias, ayudándolos a soportar mejor el tiempo de hospitalización, desdramatizando el entorno médico, ofreciendo momentos de distracción a través del humor y la risa. Para ello el proyecto se ejecuta en varios momentos donde se realiza la formación como payasos de Hospital, a través de un taller con una duración de 25 horas, donde el estudiantado debe realizar técnicas de clown de hospital, creación de materiales a utilizar en los diferentes contextos a participar (maletín de la risa y atuendos) y, por último, la participación en la comunidad como payasos de hospital, realizando al menos dos visitas como mínimo durante cada semana. Una vez formados como payasos se involucrarán con el resto de payasos de la Fundación Doctores y Doctoras de la Piñata, para la atención de personas hospitalizadas.

En este mismo sentido, en el año 2oII el Instituto Universitario AvEPANE incluye en el pensum de estudios de la Carrera Educación Especial la asignatura Pedagogía Hospitalaria. Y en el año 2012 esta institución ofrece además el diplomado de Pedagogía Hospitalaria. El diseño del diplomado y de la asignatura respondieron a un diagnóstico realizado en el año 2008 en Venezuela, sobre la base de las competencias que la UNESCO denomina «los cuatro pilares de la educación», necesarios para una educación a lo largo de la vida: Aprender a conocer, Aprender a hacer, Aprender a convivir y Aprender a ser.

Es oportuno señalar, además, que el Colegio Universitario de Psicopedagogía desde el año 20I2, en sus extensiones en las ciudades de Caracas y Valencia, a través del convenio unesco-IEsalc, imparte la asignatura Pedagogía Hospitalaria, dentro del pensum de estudios de la Carrera de Psicopedagogía en sus dos especialidades: Dificultades en el Aprendizaje y Retardo Mental, donde se hace énfasis en conocer la definición de salud disminuida, indagar sobre las enfermedades agudas y crónicas, diferenciar la percepción de la enfermedad según el desarrollo evolutivo del niño, interpretar el impacto de la enfermedad y la hospitalización en el niño y la familia, conocer los diferentes programas de atención psicoeducativa, elaboración 
de instrumentos de recolección de datos adaptados a la realidad de la hospitalización, entre otros aspectos.

Asimismo, en el año 2012 se inició el Diplomado en Pedagogía para la inclusión del niño y adolescente en situación de enfermedad y/o tratamiento, organizado por la Escuela de Educación de la Universidad Católica Andrés Bello conjuntamente con la Asociación Civil El Aula de los Sueños, cuyo propósito es favorecer la capacidad de análisis, síntesis y reflexión frente a las complejidades inherentes a la atención integral de los estudiantes hospitalizados y/o en tratamiento, para contribuir en el fortalecimiento de una visión del hombre como ser integrado por múltiples dimensiones.

Este diplomado va dirigido a docentes en ejercicio, docentes de aulas hospitalarias, personal médico y de enfermería de pediatría. También a padres, familiares o responsables que padecen alguna enfermedad, que bajo una modalidad presencial se ofertan los módulos: Construcción antropológica y ética para la vida; El hospital como un ecosistema vital; Pedagogía Hospitalaria; Atención pedagógica hospitalaria: una práctica holística; Marco legal de la atención pedagógica hospitalaria, e Interacción con la realidad pedagógica hospitalaria venezolana. El diplomado contó con dos ediciones realizadas en el año 2012 y 20I3, con una duración de I5o horas, formando un total de 28 profesionales.

Por otra parte, en el año 2014 se hizo efectivo el contrato para la creación de la Red unitwin de Pedagogía Hospitalaria entre la unesco, Universitat de Barcelona y REDLACEH, participando por Venezuela la Universidad Católica Andrés Bello y la Asociación Civil El Aula de los Sueños.

La Red UNITwin en Pedagogía Hospitalaria se ha creado con el objetivo de favorecer y generar espacios para la cooperación y el intercambio entre Universidades y Organizaciones no Gubernamentales de Latinoamérica y Europa, para el desarrollo de la Pedagogía Hospitalaria respecto a la Formación, la Investigación y la Acción, en las diferentes etapas de la vida de las personas con necesidades asistenciales.

En la actualidad y en el marco de la celebración del iı Congreso Latinoamericano y del Caribe sobre Pedagogía Hospitalaria a desarrollarse en Chile en septiembre de 20I6, esta Red Unitwin UnEsco de Pedagogía Hospitalaria llevó a cabo un Encuentro de Universidades latinoamericanas y europeas en Pedagogía Hospitalaria. Este encuentro de Universidades tuvo como objetivo presentar la Red UNITWIN UNESCO de Pedagogía Hospitalaria, a través de un workshop, donde se perseguía la interacción entre los diferentes miembros de la Red y los representantes de las universidades y diversos organismos, invitados para la construcción de conocimiento, que redunde en la comprensión del Proyecto de la Red unitwin unesco de Pedagogía Hospitalaria respecto a tres ejes: compromiso, responsabilidad y ética; investigación como base para buenas prácticas; y formación de profesionales.

Otros espacios de formación que se desarrollan en el país son los que se enumeran a continuación: pasantías interaulas hospitalarias, realización y/o participación en congresos, jornadas, foros, charlas o conversatorios en recintos educativos.

Considerando lo antes planteado, sirvan las palabras de Hermo (2013) cuando establece que es necesario y pertinente crear y diseñar un área como mención o especialización dentro de la educación, y desarrollar el perfil que requiere un docente hospitalario, cargado de recursos y herramientas para trabajar en la esencia pura de la vida con niños y adolescentes en situación diferente, porque si bien es cierto que 
estos que acuden a un hospital son pacientes, no es menos cierto que también son personas que tienen el derecho y el deber de seguir educándose.

\section{Investigación en el avance de la Pedagogía Hospitalaria}

Uno de los retos más importantes que debe afrontar la Pedagogía Hospitalaria se relaciona directamente con el hecho de estar bien fundamentada científicamente. De ahí que Lizasoáin (2016) sostiene que en Pedagogía Hospitalaria es de obligado cumplimiento centrarse no sólo en la acción, sino también en la investigación para nutrir su contenido científico.

Sobre este particular, en el país se han desarrollado diversas líneas de investigación alrededor de la Pedagogía Hospitalaria, como por ejemplo el empleo de las Tecnologías de Información y Comunicación (TIC) en el hospital, desarrollo de programas específicos de formación e intervención, estudios sobre el impacto de la enfermedad sobre el propio paciente y su grupo familiar, y análisis de aspectos legislativos, entre otros.

Asimismo, son numerosas las publicaciones realizadas por autores venezolanos en revistas nacionales e internacionales, así como la publicación de libros de texto, elaboración de tesis de pregrado y postgrado, que a su vez han generado el conferimiento de premios y distinciones nacionales. También es frecuente encontrar la participación de investigadores y docentes hospitalarios como ponentes en diferentes eventos de carácter nacional e internacional.

En este sentido, muchas de las actividades que realizan los docentes hospitalarios en el día a día es dedicarse a la investigación o, cuando menos, a algo que aporta un contenido de gran valor para la misma, a lo que hay que sacar más partido.

Si se realiza una revisión de los roles del pedagogo hospitalario, se destaca que entre sus competencias se deben incluir tanto la práctica como la investigación. Es necesario, por tanto, que el docente hospitalario realice investigaciones y publicaciones acerca de la valoración de las necesidades del niño en situación de enfermedad y de su familia, así como investigaciones relacionadas con la intervención educativa, evaluando diferentes variables en determinadas situaciones, a través del empleo y aplicación de programas y metodologías variadas.

\section{Programa Aulas Fundación Telefónica en Hospitales}

Desde el año 2007, la Fundación Telefónica lleva a cabo en Venezuela el proyecto Aulas Fundación Telefónica en Hospitales, iniciativa global que promueve el uso educativo de las Tecnologías de Información y Comunicación (TIC) y sus buenas prácticas en espacios de aprendizaje desarrollados en hospitales, donde a través de equipos tecnológicos, niños y adolescentes hospitalizados tienen la oportunidad de dar continuidad a la formación escolar participando en programas que esta fundación crea especialmente para ellos. Su implementación viene acompañada de un programa de capacitación continua, que incorpora a los docentes en el uso de las Tic y su aplicación educativa.

Este programa consiste en la instalación y funcionamiento de áreas especialmente habilitadas para brindar atención educativa a niños y adolescentes durante la estancia 
en centros de salud, de tal manera que puedan continuar su formación mediante el uso pedagógico de las Tic. El propósito del programa es brindar atención educativa a los niños y adolescentes que, en edad de escolarización, presentan una situación de enfermedad que requieren hospitalización más o menos prolongada.

Según la Fundación Telefónica (2014) este programa se centra además en el derecho de todos los niños y adolescentes hospitalizados a continuar su proceso de aprendizaje, fortaleciendo sus capacidades de expresión, comprensión, razonamiento y valores sociales, acompañando todos estos procesos con el uso las TIC como herramienta fundamental de enseñanza y aprendizaje, principalmente bajo el diseño de una metodología lúdica.

En la actualidad, la Fundación Telefónica cuenta con trece (13) aulas hospitalarias entre fijas y móviles, que funcionan en áreas pediátricas de siete (7) centros de salud del país, atendiéndose a lo largo de estos años a 25.000 pequeños pacientes aproximadamente.

Otro de los proyectos de la Fundación Telefónica en Venezuela es la instalación de Salas Digitales Movistar. Las Salas Digitales son espacios multiuso donde se emplean recursos TIC para favorecer el proceso de aprendizaje, interacción, creación y conexión. Entre los años 2014 a 2016 se han instalado en el país cincuenta (50) Salas Digitales, algunas de ellas en entornos hospitalarios. Las Salas Digitales se encuentran distribuidas en los siguientes hospitales del país: Hospital Universitario de Pediatría «Dr. Agustín Zubillaga», Hospital de Especialidades Pediátricas de Maracaibo, Instituto Oncológico «Luis Razetti», Hospital Militar «Dr. Carlos Arvelo» (FUNDACARDIN) y en el Hospital de Niños «José Manuel de los Ríos».

\section{Programa de Atención Hospitalaria}

En junio de 2012, la Fundación Musical Simón Bolívar creó el Programa de Atención Hospitalaria (PAH) para impartir formación musical a niños y jóvenes en hospitales. Con ello, el Sistema Nacional de Orquestas y Coros Juveniles e Infantiles de Venezuela busca beneficiar a pacientes que padecen enfermedades que les mantienen por largos períodos en los centros de salud.

«El Sistema», enmarcado en el modelo formativo humanista y de inclusión social, busca atender a niños y niñas en edades comprendidas entre los 6 a I8 años de edad, que estén ingresados en instituciones médicas del país, ofreciéndoles clases de música, específicamente en las cátedras de Cuatro, Guitarra, Campanas de Colores, Percusión y Violín, con el fin de acompañarlos en su convalecencia, mediante el estudio de la música y la atención directa y personalizada del docente, llegando hasta la misma habitación del paciente en caso de que el niño tenga movilidad limitada a causa de su patología.

En el Distrito Capital se desarrolla el Programa en el Hospital de Niños «Dr. José Manuel de los Ríos», Hospital Militar «Dr. Carlos Arvelo», Hospital Cardiológico Infantil Latinoamericano «Dr. Gilberto Rodríguez Ochoa», Casa Hogar «Madre Teresa de Calcuta» y próximamente en la Casa Hogar «Ronald McDonalds» y el Hospital Ortopédico Infantil; y en el interior del país en el Hospital Central «Dr. Miguel Oraá» de Guanare, Estado Portuguesa.

El propósito del Programa es lograr la integración e inclusión social del participante del PAH, a través del acompañamiento formativo durante y después de su 
proceso de recuperación, utilizando la música como herramienta de proyección y motivación personal. Entre los objetivos que persiguen, se pueden mencionar:

- Acompañar en el proceso de rehabilitación a los niños y adolescentes ingresados en hospitales, fomentando en ellos la esperanza a través de la música.

- Impartir clases de música, en las cátedras de Cuatro, Guitarra, Campanas de Colores, Percusión, Xilófono y Violín a los niños y adolescentes hospitalizados.

- Organizar repertorios musicales adecuados para el niño o adolescente, acordes a la edad, estado anímico y proceso evolutivo de la enfermedad.

- Realizar recitales y conciertos mensuales, dentro y fuera de las instalaciones del Hospital, demostrando el desempeño alcanzado por el alumno paciente.

- Insertar al niño y adolescente en los programas formales de estudios musicales, ubicándolo en el Núcleo del Sistema de Orquestas y Coros Juveniles e Infantiles de Venezuela más cercano a su comunidad, una vez reciba el alta médica, para que allí pueda continuar de manera efectiva su crecimiento en el área musical.

El Programa cuenta con doce (I2) docentes encargados de las diferentes cátedras, y desde el año 2012 ha beneficiado a más de 2.000 integrantes pacientes, atendiendo en promedio a 142 niños y adolescentes mensualmente, así como también participan en promedio veinte (20) profesionales de la salud en las actividades artísticas.

De esta manera, la música se convierte en herramienta terapéutica y en esperanza de sanación para muchos jóvenes y niños. Es a través del contacto con la música como este programa permite aminorar en la población beneficiada los grados de ansiedad y estrés que cada patología ocasiona, lo cual favorece, además, la relación familia-paciente-médico.

\section{Proyectos de Atención Integral a Niños Pacientes: SaludArte}

La Asociación Civil Venezolana Círculos de Investigaciones y Estudios con el Psicoanálisis (CIEP) a través de un proyecto de investigación acción denominado SaludArte desarrolla desde el año 2002 una línea de trabajo dirigida a niños hospitalizados, siendo uno de sus objetivos la difusión de los derechos de niños y adolescentes en condición de hospitalización. Esta línea de investigación, según Izaguirre (2007), vincula el psicoanálisis con otras disciplinas tales como educación, medicina, sociología, psicología, estudios de género, arte, derecho, entre otras.

A través de las actividades que desarrollan buscan establecer relaciones profesionales con diversas instituciones para niños y adolescentes en centros de salud y hospitales, con el propósito de estimular la comunicación de niños y adolescentes con su familia y el entorno, proporcionar entretenimiento para aquellos que están limitados por su condición física, romper la monotonía del ambiente hospitalario, ayudar en la reducción de síntomas emocionales producto de la enfermedad y la hospitalización, mantener a los niños y adolescentes hospitalizados cognitivamente activos favoreciendo su rehabilitación física y emocional.

A continuación, se describirá brevemente cada uno de los proyectos que esta asociación ha puesto en marcha:

Contarte, leyendo en el hospital. Tiene como objetivo brindar a los niños y sus padres una herramienta que, a través de la lectura, favorezca la comunicación y ayude a 
reducir los síntomas emocionales producto de la experiencia de hospitalización. Este proyecto permite la distribución de manuales interactivos impresos y además se realizan talleres para la inducción y presentación audiovisual de los Derechos de niños pacientes. Entre los manuales se encuentran: Manual del Niño Paciente: Hospitalización y Cirngía, Manual del Niño Paciente: Enfermedades Oncológicas, Manual para Padres y Voluntarios del Niño Paciente y Cartilla de Derechos y Deberes del Niño Paciente.

Dentro de este proyecto, se encuentra además la revista La Piñata, una publicación bimensual creada especialmente para niños en situación de enfermedad hospitalizados, distribuida gratuitamente en instituciones hospitalarias públicas de todo el país, la cual recibió el Premio Rafael Ángel García en el año 2006. Sacar una sonrisa entre un mar de llantos es el principal objetivo de esta publicación. Esta revista combina elementos de pedagogía y juego, con el fin de contribuir a la elevación de la calidad de vida de niños y adolescentes pacientes, así como su grupo familiar, durante su permanencia en instituciones hospitalarias. En esta publicación además se les ofrece a niños hospitalizados la oportunidad de expresar y recrear sus emociones y pensamientos vinculados con su experiencia en la enfermedad, lo cual influye positivamente en su desarrollo psicológico y en una mejor adaptación a las condiciones de esa patología. Todos los contenidos de la revista La Piñata están enmarcados en la Ley Orgánica para la Protección de Niños, Niñas y Adolescentes (LOPNA) de Venezuela, a través de contenidos especiales que constituyen herramientas que activan la participación de los pequeños.

Recrearte, jugando en el hospital. Tiene como objetivo entretener al pequeño paciente con arte, juegos y manualidades durante su permanencia en el hospital, desarrollando actividades de expresión plástica (dibujo, pintura, modelado, costura, fabricación de juguetes, entre otras); actividades de expresión dramática y corporal (títeres, teatro, magia, expresión corporal y técnicas de relajación); actividades de expresión musical (canciones tradicionales, instrumentos musicales e iniciación musical); Doctores de la Piñata (un grupo de payasos de hospital brindan compañía y entretenimiento a niños y adolescentes hospitalizados). Este proyecto fue merecedor del Premio fama en el año 2003.

Mirarte, audiovisuales. Con el objetivo de sensibilizar masivamente el entorno social respecto a las necesidades físicas y afectivas generadas en niños y adolescentes hospitalizados y su entorno familiar por el cambio introducido en sus vidas por la aparición de una enfermedad, y sugerir respuestas para que las consecuencias sean sobrellevadas en las mejores condiciones posibles, contribuyendo en la mejora de la calidad de vida de estos niños y sus familias, han diseñado una serie de televisión de dieciséis (I6) capítulos, titulada Sala 404 Historias de Niños Pacientes, proyectada en el canal de televisión vive TV, donde se describen los Derechos del Niño Paciente, que también se encuentran disponibles en DVD para trabajar directamente con los niños en el hospital, así como Consejos para Padres y Voluntarios del Niño Paciente. Este proyecto recibió el Premio Metropolitano de Periodismo Aníbal Nazoa en 2007, y el Premio Rafael Ángel García en el año 2008.

Pintarte, ambientando mi hospital. Nace con el objetivo de transformar los espacios de hospitalización infantil en lugares de asistencia médica acogedores, ligados a la creación artística y a la información. Cuenta con dos componentes: Ambientación del Hospital y Salud para mi Hospital.

En la actualidad el CIEP se encuentra elaborando el Protocolo de Atención dirigido al personal sanitario, desde la perspectiva del Buen Trato a niños y adolescentes, en 
el contexto de la atención en salud, a solicitud de la Defensoría del Pueblo del Estado Venezolano.

\section{Consideraciones finales}

La elaboración de este documento ha posibilitado tener una visión general de lo que se ha hecho y se está haciendo en Pedagogía Hospitalaria en Venezuela. Por lo que permite afirmar que, a pesar de lo reciente de esta modalidad, se han obtenido avances significativos. Entre ellos se puede señalar que existen 78 espacios a lo largo del país que atienden a niños y adolescentes en situación de enfermedad o en tratamiento, velando por el cumplimiento del derecho a ser educados y constituyendo una herramienta valiosa que permite la mejora en la calidad de vida y la salud integral de estos niños, adolescentes y sus familias.

Asimismo se considera que la Pedagogía Hospitalaria en Venezuela se ha desarrollado positivamente en forma progresiva, sin embargo, existen todavía necesidades, y aspectos a mejorar en lo que se refiere al rol de las instituciones implicadas en la generación de políticas, a través de los mecanismos legales o reglamentarios pertinentes; por tanto, se requieren acciones coordinadas y coherentes en todas las instancias gubernamentales y no gubernamentales dirigidas al fortalecimiento de la Pedagogía Hospitalaria en el país.

En este sentido, Hermo (2013) sostiene que sería oportuno desarrollar un marco legal en Venezuela que proteja los derechos de los niños en situación de hospitalización o convalecencia prolongada e incorporar, paulatinamente, las instituciones de educación superior para llevar a cabo proyectos en esta área de atención en la educación, así como también la difusión y el conocimiento de la Pedagogía Hospitalaria en el sistema regular de educación, a la par de proyectar campañas comunicacionales para sensibilizar e informar en torno al Derecho a la Educación de los niños y adolescentes que presenten condiciones específicas de salud.

Existe igualmente la necesidad de establecer una más estrecha relación con la escuela de origen, ya que resulta de vital importancia que ésta mantenga la relación con el niño en situación de enfermedad en forma permanente a través de la familia y los docentes del aula hospitalaria, con la finalidad de programar su posterior inserción, que debería contemplar lo académico, lo social y todo lo que haga referencia a su situación de enfermedad, ya que la realidad muestra que un gran número de escuelas de origen no realiza un seguimiento, lo que conlleva pensar en la falta de conocimiento de la realidad de las Aulas Hospitalarias en el ámbito educativo en general.

Se constata a su vez que falta atención educativa a niños en situación de enfermedad que debe irse cubriendo con nuevos establecimientos a lo largo del territorio nacional, así como atención educativa domiciliaria. Así, sería deseable que en cada proyecto de creación de hospitales con atención pediátrica se cuente con el servicio de Aula Hospitalaria. También se observa la necesidad de una formación docente especializada tanto para los profesionales a cargo de las Aulas Hospitalarias como para los docentes de escuelas regulares. Para esto se ve la necesidad de acercar la praxis al mundo académico, mediante la sistematización del quehacer pedagógico hospitalario, la investigación y la creación y/o prosecución de programas o planes sobre esta modalidad educativa en las carreras de educación superior universitaria. 
Por otra parte, las experiencias descritas en este trabajo acerca de los diferentes programas que se desarrollan en los hospitales del país dan cuenta del creciente interés de la utilización de medios tecnológicos, la música, las actividades artísticas, entre otros; como una posibilidad para enriquecer el proceso educativo de los niños y adolescentes hospitalizados, para involucrarlos de forma más directa con la escuela, cuestión que es una necesidad inminente para su crecimiento físico, intelectual y emocional. Cabe destacar que cada vez se considera más oportuno en esta modalidad educativa el uso de estos recursos como una necesidad del niño y adolescente en situación de enfermedad.

Sobre la base de las consideraciones anteriores, estos desafíos planteados impulsan a movilizar a los implicados en esta modalidad educativa en el país a seguir trabajando en red con el fin de consensuar criterios fundamentales, desde las voluntades públicas y privadas, para brindar una atención pedagógica acorde a las necesidades de los niños y adolescentes en situación de enfermedad y/o tratamiento y sus familias.

\section{Bibliografía}

Cardone, P. y Monsalve, C. (2010) Pedagogía Hospitalaria. Una propuesta educativa. Caracas: FEDUPEL.

Fundación Musical Simón Bolívar. Programa de Atención Hospitalaria. Recuperado el 26 de agosto de 20I6, de https://fundamusical.org.ve/educacion/programa-de-atencion-hospitalaria/.

Fundación Telefónica (20I4) Sistematización de buenas prácticas. Atención integral del niño, niña y adolescente en Aulas Fundación Telefónica en Hospitales. Caracas: Fundación Telefónica Venezuela.

Hermo, C. (2013) El perfil del docente que labora en espacios educativos en el contexto hospitalario. Revista Latidos, $3,30$.

Izaguirre, M. (2007) Saludarte: Proyectos de Atención Integral a Niños Pacientes. Recuperado el 06 de septiembre de 2016, de http://www.pedagogiahospitalaria.net/jornadas/2007/ ponencias/Maria_Antonieta_Izaguirre.pdf.

John, Y. (2006) La Pedagogía Hospitalaria en el Marco del Sistema Educativo Bolivariano. Caracas: Ministerio de Educación y Deportes.

Ley Marco para la Pedagogía Hospitalaria en América Latina y el Caribe (20r4) Comisión de Educación, Cultura, Ciencia, Tecnología y Comunicación. Chile.

Ley Orgánica para la Protección del niño, niña y del adolescente (1998) Gaceta Oficial de la República de Venezuela, n. ${ }^{\circ}$ 5266, 2 de octubre de 1998 . Venezuela.

Lizardo, J. (2010) Programa de la asignatura electiva Abordaje pedagógico del infante en situación de hospitalización. Caracas: Universidad Pedagógica Experimental Libertador, Instituto Pedagógico de Caracas.

LizasoÁrn, O. (20I6) Pedagogía Hospitalaria. Guía para la atención psicoeducativa del alumno enfermo. Madrid: Síntesis.

Rodríguez, L. (2012) Programa de la asignatura electiva Pedagogía Hospitalaria. Caracas: Universidad Central de Venezuela, Escuela de Educación.

Serradas, M. (2008) Los derechos de los niños hospitalizados: Un compromiso ineludible. Revista Archivos Venezolanos de Puericultura y Pediatría, 7I (2), 59-67.

Universidad Pedagógica Experimental Libertador. Instituto de Mejoramiento Profesional del Magisterio. Ministerio de Educación (i998) Conceptualización y Politica de la atención educativa de las personas con impedimentos físicos. Caracas: Autor.

Violant, V.; Molina, M. y Pastor, C. (20II) Pedagogía Hospitalaria. Bases para la atención integral. Barcelona: Laertes. 